J. Korean Math. Soc. 51 (2014), No. 1, pp. 113-123

http://dx.doi.org/10.4134/JKMS.2014.51.1.113

\title{
EXTREME PRESERVERS OF TERM RANK INEQUALITIES OVER NONBINARY BOOLEAN SEMIRING
}

\author{
LeRoy B. Beasley, Seong-Hee Heo, and Seok-Zun Song
}

\begin{abstract}
The term rank of a matrix $A$ over a semiring $\mathcal{S}$ is the least number of lines (rows or columns) needed to include all the nonzero entries in $A$. In this paper, we characterize linear operators that preserve the sets of matrix ordered pairs which satisfy extremal properties with respect to term rank inequalities of matrices over nonbinary Boolean semirings.
\end{abstract}

\section{Introduction}

There are many papers on linear operators that preserve some properties of matrices ([2]-[12]). We call such a topic of research "Linear Preserver Problems". These linear preserver problems have been studied for various characterizations of matrices and linear operators during the last century. Beasley and Guterman ([1]) investigated linear preservers of rank inequalities of matrices over semirings without zero-divisors. And they characterized the equality cases for some inequalities of matrix functions over semirings in [2]. These characterization problems are open even over fields (see [3]). The structure of matrix varieties which arise as extremal cases of these inequalities is far from being understood over fields, as well as over semirings. A usual way to generate elements of such a variety is to find a pair of matrices which belongs to it and to act on this pair by various linear operators that preserve this variety. The investigation of the corresponding problems over semirings for the column rank function was done in [3]. The complete classification of linear operators that preserve equality cases in matrix inequalities over fields was obtained in [4]. For details on linear operators preserving matrix invariants one can see [9] and [10]. Almost all research on linear preserver problems over semirings have dealt with those semirings without zero-divisors to avoid the difficulties of multiplication arithmetic for the elements in those semirings ([2]-[7]). However, in the

Received January 8, 2013; Revised September 3, 2013.

2010 Mathematics Subject Classification. Primary 15A86, 15A03, 15 B34.

Key words and phrases. term rank, linear operator, nonbinary Boolean semiring.

This research was supported by Basic Science Research Program through the National Research Foundation of Korea(NRF) funded by the Ministry of Education, Science and Technology(No. 2012R1A1A2042193). 
case of a nonbinary Boolean semiring, all elements except 0 and 1 in nonbinary Boolean semirings are zero-divisors. So there are few results on linear preserver problems for matrices over nonbinary Boolean semirings ([11]-[12]). Kirkland and Pullman characterized the linear operators that preserve the rank of matrices over nonbinary Boolean semirings in [8]. Since the term rank is an important matrix function, there are many research papers on term rank and its preservers $([5]-[7])$.

In this paper, we characterize the linear operators that preserve the sets of matrix ordered pairs which satisfy extremal properties with respect to term rank inequalities of matrices over nonbinary Boolean semirings.

\section{Preliminaries and basic results}

Definition 2.1. A semiring $\mathcal{S}$ consists of a set $\mathcal{S}$ with two binary operations, addition and multiplication, such that:

. $\mathcal{S}$ is an Abelian monoid under addition (the identity is denoted by 0 );

- $\mathcal{S}$ is a monoid under multiplication (the identity is denoted by $1,1 \neq 0$ );

- multiplication is distributive over addition on both sides;

$\cdot s 0=0 s=0$ for all $s \in \mathcal{S}$.

Definition 2.2. A semiring $\mathcal{S}$ is called antinegative if the zero element is the only element with an additive inverse.

Definition 2.3. A semiring $\mathcal{S}$ is called a Boolean semiring if $\mathcal{S}$ is equivalent to a set of subsets of a given set $\mathcal{Y}$, the sum of two subsets is their union, and the product is their intersection. The zero element is the empty set and the identity element is the whole set $\mathcal{Y}$.

Let $S_{k}=\left\{a_{1}, a_{2}, \ldots, a_{k}\right\}$ be a set of $k$-elements, $\mathcal{P}\left(S_{k}\right)$ be the set of all subsets of $S_{k}$ and $\mathbb{B}_{k}$ be the Boolean semiring of all subsets of $S_{k}=\left\{a_{1}, a_{2}, \ldots, a_{k}\right\}$, $\mathcal{P}\left(S_{k}\right)$. Let $\mathbb{S}_{k}$ be a Boolean semiring of subsets of $S_{k}=\left\{a_{1}, a_{2}, \ldots, a_{k}\right\}$, that is a subset of $\mathcal{P}\left(S_{k}\right)$. It is straightforward to see that a Boolean semiring $\mathbb{S}_{k}$ is a commutative and antinegative semiring. If $\mathbb{S}_{k}$ consists of only the empty subset and $S_{k}$, then it is called a binary Boolean semiring. If $\mathbb{S}_{k}$ is not a binary Boolean semiring, then it is called a nonbinary Boolean semiring. Let $\mathbb{M}_{m, n}\left(\mathbb{S}_{k}\right)$ denote the set of $m \times n$ matrices with entries from the Boolean semiring $\mathbb{S}_{k}$. If $m=n$, we use the notation $\mathbb{M}_{n}\left(\mathbb{S}_{k}\right)$ instead of $\mathbb{M}_{n, n}\left(\mathbb{S}_{k}\right)$.

Throughout the paper, we assume that $m \leq n$ and $\mathbb{S}_{k}$ denotes a nonbinary Boolean semiring, which contains at least 3 elements. The matrix $I_{n}$ is the $n \times n$ identity matrix, $J_{m, n}$ is the $m \times n$ matrix of all ones and $O_{m, n}$ is the $m \times n$ zero matrix. We omit the subscripts when the order is obvious from the context and we write $I, J$ and $O$, respectively. The matrix $E_{i, j}$, which is called a cell, denotes the matrix with exactly one nonzero entry, that being a one in the $(i, j)^{t h}$ entry. Let $R_{i}$ denote the matrix whose $i^{t h}$ row is all ones and is zero elsewhere, and $C_{j}$ denote the matrix whose $j^{\text {th }}$ column is all ones and is zero elsewhere. 
Definition 2.4. Let $\mathbb{S}_{k}$ be a nonbinary Boolean semiring. An operator $T$ : $\mathbb{M}_{m, n}\left(\mathbb{S}_{k}\right) \rightarrow \mathbb{M}_{m, n}\left(\mathbb{S}_{k}\right)$ is called linear if it satisfies $T(X+Y)=T(X)+T(Y)$ and $T(\alpha X)=\alpha T(X)$ for all $X, Y \in \mathbb{M}_{m, n}\left(\mathbb{S}_{k}\right)$ and $\alpha \in \mathbb{S}_{k}$.

Definition 2.5. A line of a matrix $A$ is a row or a column of the matrix $A$.

Definition 2.6. The matrix $A \in \mathbb{M}_{m, n}\left(\mathbb{S}_{k}\right)$ is said to be of term rank $k(t(A)=$ $k$ ) if the least number of lines needed to include all nonzero elements of $A$ is equal to $k$. Let us denote by $c(A)$ the least number of columns needed to include all nonzero elements of $A$ and by $r(A)$ the least number of rows needed to include all nonzero elements of $A$.

Definition 2.7. The matrix $A \in \mathbb{M}_{m, n}\left(\mathbb{S}_{k}\right)$ is said to be of Boolean rank $r$ if there exist matrices $B \in \mathbb{M}_{m, r}\left(\mathbb{S}_{k}\right)$ and $C \in \mathbb{M}_{r, n}\left(\mathbb{S}_{k}\right)$ such that $A=B C$ and $r$ is the smallest positive integer such that such a factorization exists. We denote $b(A)=r$.

By definition, the unique matrix with Boolean rank equal to 0 is the zero matrix $O$.

Arithmetic properties of term rank of Boolean matrices are restricted by the following list of inequalities established in [1]:

(1) $t(A+B) \leq t(A)+t(B)$;

(2) $t(A+B) \geq \max \{t(A), t(B)\}$;

(3) $t(A B) \leq \min \{r(A), c(B)\}$

(4) $t(A B) \geq t(A)+t(B)-n$;

(5) If $\mathcal{S}$ is a subsemiring of positive reals, then $\rho(A B)+\rho(B C) \leq t(A B C)+$ $t(B)$, where $\rho(X)$ is the real rank of the matrix $X$.

Below, we use the following notation to denote sets of Boolean matrices that arise as extremal cases in the inequalities listed above:

$$
\begin{aligned}
& \mathcal{T}_{s a}\left(\mathbb{S}_{k}\right)=\left\{(X, Y) \in \mathbb{M}_{m, n}\left(\mathbb{S}_{k}\right)^{2} \mid t(X+Y)=t(X)+t(Y)\right\} ; \\
& \mathcal{T}_{s m}\left(\mathbb{S}_{k}\right)=\left\{(X, Y) \in \mathbb{M}_{m, n}\left(\mathbb{S}_{k}\right)^{2} \mid t(X+Y)=\max \{t(X), t(Y)\}\right\} ; \\
& \mathcal{T}_{m n}\left(\mathbb{S}_{k}\right)=\left\{(X, Y) \in \mathbb{M}_{n}\left(\mathbb{S}_{k}\right)^{2} \mid t(X Y)=\min \{r(X), c(Y)\}\right\} ; \\
& \mathcal{T}_{m a}\left(\mathbb{S}_{k}\right)=\left\{(X, Y) \in \mathbb{M}_{n}\left(\mathbb{S}_{k}\right)^{2} \mid t(X Y)=t(X)+t(Y)-n\right\} ; \\
& \mathcal{T}_{m t}\left(\mathbb{S}_{k}\right)=\left\{(X, Y, Z) \in \mathbb{M}_{n}\left(\mathbb{S}_{k}\right)^{3} \mid t(X Y Z)+t(Y)=t(X Y)+t(Y Z)\right\} .
\end{aligned}
$$

Definition 2.8. Let $S$ be any semiring. We say an operator, $T: \mathcal{M}_{m, n}(S) \rightarrow$ $\mathcal{M}_{m, n}(S)$, preserves a set $\mathcal{P}$ if $X \in \mathcal{P}$ implies that $T(X) \in \mathcal{P}$, or, if $\mathcal{P}$ is a set of ordered pairs (triples), provided that $(X, Y) \in \mathcal{P}((X, Y, Z) \in \mathcal{P})$ implies $(T(X), T(Y)) \in \mathcal{P}((T(X), T(Y), T(Z)) \in \mathcal{P}$, respectively).

Definition 2.9. The matrix $X \circ Y$ denotes the Hadamard or Schur product, i.e., the $(i, j)$ entry of $X \circ Y$ is $x_{i, j} y_{i, j}$.

Definition 2.10. Let $S$ be any semiring. An operator $T: \mathcal{M}_{m, n}(S) \rightarrow$ $\mathcal{M}_{m, n}(S)$ strongly preserves the set $\mathcal{P}$ if $X \in \mathcal{P}$ if and only if $T(X) \in \mathcal{P}$, or, if $\mathcal{P}$ is a set of ordered pairs (triples), provided that $(X, Y) \in \mathcal{P}((X, Y, Z) \in \mathcal{P})$ if and only if $(T(X), T(Y)) \in \mathcal{P}((T(X), T(Y), T(Z)) \in \mathcal{P}$, respectively). 
Definition 2.11. Let $S$ be any semiring. An operator $T: \mathcal{M}_{m, n}(S) \rightarrow$ $\mathcal{M}_{m, n}(S)$ is called a $(P, Q, B)$-operator if there exist permutation matrices $P$ and $Q$, and a matrix $B \in \mathbb{M}_{m, n}(\mathcal{S})$ with no zero entries, such that

$$
T(X)=P(X \circ B) Q
$$

for all $X \in \mathbb{M}_{m, n}(\mathcal{S})$, or, if $m=n$,

$$
T(X)=P(X \circ B)^{t} Q
$$

for all $X \in \mathbb{M}_{m, n}(\mathcal{S})$, where $X^{t}$ denotes the transpose of $X$. Operator of the form (2.1) is called non-transposing $(P, Q, B)$-operator; operators of the form (2.2) is called transposing $(P, Q, B)$-operator. A $(P, Q, B)$-operator is called a $(P, Q)$-operator if $B=J$, the matrix of all ones.

In [4] linear preservers of extremal cases of classical matrix inequalities over fields were characterized. On the other hand, linear preservers for various rank functions over semirings have been the object of much study during the last 30 years, see for example [2]-[12]. In particular term rank was investigated in the last years, see for example $[5,6,7]$. The aim of the present paper is to classify linear operators that preserve pairs of matrices that attain extreme cases in the above term rank inequalities 1-5.

Definition 2.12. We say that the matrix $A$ dominates the matrix $B$ if and only if $b_{i, j} \neq 0$ implies that $a_{i, j} \neq 0$, and we write $A \geq B$ or $B \leq A$.

We begin with some basic results which can be found in [11] and [12]

Theorem 2.13 ([11, Theorem 2.1]). Let $T: \mathbb{M}_{m, n}\left(\mathbb{S}_{k}\right) \rightarrow \mathbb{M}_{m, n}\left(\mathbb{S}_{k}\right)$ be a linear operator. Then the following conditions are equivalent:

(a) $T$ is bijective;

(b) $T$ is surjective;

(c) $T$ is injective;

(d) there exists a permutation $\sigma$ on $\{(i, j) \mid i=1,2, \ldots, m ; j=1,2, \ldots, n\}$ such that $T\left(E_{i, j}\right)=E_{\sigma(i, j)}$ for all $1 \leq i \leq m$ and $1 \leq j \leq n$.

Lemma 2.14. Let $T: \mathbb{M}_{m, n}\left(\mathbb{S}_{k}\right) \rightarrow \mathbb{M}_{m, n}\left(\mathbb{S}_{k}\right)$ be a linear operator which maps lines to lines and $T$ be defined by the rule $T\left(E_{i, j}\right)=b_{i, j} E_{\sigma(i, j)}$, where $\sigma$ is a permutation on the set $\{(i, j) \mid i=1,2, \ldots, m ; j=1,2, \ldots, n\}$ and $b_{i, j}$ is a nonzero element of $\mathbb{S}_{k}$ which is not a zero divisor. Then $T$ is a $(P, Q, B)$ operator where $B$ has no entry that is either zero or a zero divisor.

Proof. For the case $\mathbb{S}_{k}=\mathbb{B}_{k}$ see [12, Lemma 2.4]. In the more general case, the same proof applies with the observation that a line is mapped into a weighted line (not all entries are 1 , but rather nonzero).

Example 2.15. Consider the linear operator $T: \mathbb{M}_{3,3}\left(\mathbb{B}_{3}\right) \rightarrow \mathbb{M}_{3,3}\left(\mathbb{B}_{3}\right)$ defined by $T(X)=X \circ B$ for all $X \in \mathbb{M}_{3,3}\left(\mathbb{B}_{3}\right)$ with $\mathbb{B}_{3}=\mathcal{P}(\{a, b, c\})$. Then $t(B)=3$ and $b(B)=1$ but we show that $T$ does not preserve the term rank if $B \neq J$. 


$$
\begin{gathered}
\text { For, let } X=\left[\begin{array}{ccc}
\{a, b\} & \{a, b, c\} & \{a, b\} \\
\{a, c\} & \{a, c\} & \{a, b\} \\
\{a\} & \{b, c\} & \{a, b\}
\end{array}\right] \text { and } B=\left[\begin{array}{ccc}
\{a\} & \{b\} & \{c\} \\
\{a\} & \{b\} & \{c\} \\
\{a\} & \{b\} & \{c\}
\end{array}\right] \text {. Then } t(X)=3 \text {, but } \\
T(X)=X \circ B=\left[\begin{array}{ccc}
\{a\} & \{b\} & 0 \\
\{a\} & 0 & 0 \\
\{a\} & \{b\} & 0
\end{array}\right] .
\end{gathered}
$$

That is, $t(T(X))=t(X \circ B)=2 \neq 3=t(X)$. Thus $t(B)=3$ but $T$ does not preserve the term rank since every nonzero nonunit entry of $B$ is a zero-divisor.

In fact, the following is true:

Theorem 2.16. Let $S$ be any semiring and $T: \mathcal{M}_{m, n}(S) \rightarrow \mathcal{M}_{m, n}(S)$ be the linear operator defined by $T(X)=X \circ B$ for some $B \in \mathbb{M}_{m, n}(\mathcal{S})$. If $B$ has entries which are zero or zero-divisors, then $T$ does not preserve any term rank.

Proof. Let $1 \leq r \leq \min \{m, n\}$. If $b_{i, j}$ is zero, or a zero-divisor, let $q \in S$ be chosen so that $b_{i, j} q=0$. Let $X$ be a matrix with exactly $r$ nonzero entries whose term rank is $r$ and such that $x_{i, j}=q$. Then $T(X)$ has at most $r-1$ nonzero entries and hence has term rank at most $r-1$. Thus, $T$ does not preserve term rank $r$.

We now require that the matrix $B$ for any $(P, Q, B)$-operator consists only of nonzero entries which are not zero-divisors.

\section{Extremes preservers of term rank over nonbinary Boolean semirings}

In this section, we characterize the linear operators that preserve the extreme set of matrix pairs, which are driven from the inequalities of the term ranks of matrices over nonbinary Boolean semiring.

We begin with lemmas.

Lemma 3.1. Suppose $\mathbb{M}$ is any finite set and $f: \mathbb{M} \rightarrow \mathbb{M}$ is a function. Then there exists an integer $p>1$ such that $f^{p}$ is idempotent.

Proof. There are only finitely many functions taking $\mathbb{M}$ to $\mathbb{M}$. Therefore the sequence $\left\{f, f^{2}, \ldots, f^{n}, \ldots\right\}$ cycles after $n$ is sufficiently large. That is, there exist integers $N>1$ and $d>1$ such that for all $m>N$ and $n>N, f^{m}=f^{n}$ if $m=n(\bmod d)$. Let $p=N d$; then $f^{p}=f^{2 p}$.

Note that the term rank depends only on which entries are non zero, not on which nonzero element they are. Thus if $B$ has no zero entries and no zero-divisor entries, then $t(X \circ B)=t(X)$.

Lemma 3.2. Let $\mathbb{S}_{k}$ be a nonbinary Boolean semiring, and $T: \mathbb{M}_{m, n}\left(\mathbb{S}_{k}\right) \rightarrow$ $\mathbb{M}_{m, n}\left(\mathbb{S}_{k}\right)$ be a $(P, Q, B)$-operator. Then $T$ preserves all term ranks. 
Proof. Assume that $T$ is a $(P, Q, B)$-operator. For any $X \in \mathbb{M}_{m, n}\left(\mathbb{S}_{k}\right)$, we have

$$
t(T(X))=t(P(X \circ B) Q)=t(X \circ B)=t(X)
$$

or if $m=n$,

$$
t(T(X))=t\left(P(X \circ B)^{t} Q\right)=t\left((X \circ B)^{t}\right)=t(X) .
$$

Hence any $(P, Q, B)$-operator preserves all term ranks.

Theorem 3.3. Let $\mathbb{S}_{k}$ be a nonbinary Boolean semiring, $T: \mathbb{M}_{m, n}\left(\mathbb{S}_{k}\right) \rightarrow$ $\mathbb{M}_{m, n}\left(\mathbb{S}_{k}\right)$ be a surjective linear map. Then $T$ preserves the set $\mathcal{T}_{\text {sa }}\left(\mathbb{S}_{k}\right)$ if and only if $T$ is a $(P, Q)$-operator, where $P$ and $Q$ are permutation matrices of appropriate sizes.

Proof. $(\Leftarrow)$ Assume that $T$ is a $(P, Q)$-operator. Then $T$ preserves all term ranks by Lemma 3.2. Therefore for any $(X, Y) \in \mathcal{T}_{s a}\left(\mathbb{S}_{k}\right)$, we have $t(X+Y)=$ $t(X)+t(Y)$. Thus

$$
\begin{aligned}
t(T(X)+T(Y)) & =t(T(X+Y))=t(X+Y) \\
& =t(X)+t(Y)=t(T(X))+t(T(Y)) .
\end{aligned}
$$

Hence $(P, Q)$-operators preserve the set $\mathcal{T}_{s a}\left(\mathbb{S}_{k}\right)$.

$(\Rightarrow)$ If $T$ is surjective, then by Theorem 2.13 we have that $T\left(E_{i, j}\right)=E_{\sigma(i, j)}$ for all $i, j, 1 \leq i \leq m, 1 \leq j \leq n$, where $\sigma$ is a permutation on the set of pairs $(i, j)$.

Let us show that $T$ maps lines to lines. Suppose that the images of two cells are in the same line, but the cells are not, say $E_{i, j}, E_{k, l}$ are the cells such that $t\left(E_{i, j}+E_{k, l}\right)=2$ and $t\left(T\left(E_{i, j}+E_{k, l}\right)\right)=1$. Then $\left(E_{i, j}, E_{k, l}\right) \in \mathcal{T}_{s a}\left(\mathbb{S}_{k}\right)$ but $\left(T\left(E_{i, j}\right), T\left(E_{k, l}\right)\right) \notin \mathcal{T}_{s a}\left(\mathbb{S}_{k}\right)$, a contradiction. Thus $T$ maps lines to lines. Thus by Lemma $2.14 T$ is a $(P, Q)$-operator where $P$ and $Q$ are permutation matrices of appropriate sizes.

Now we can improve Theorem 3.3 by removing the surjectivity assumption:

Theorem 3.4. Let $\mathbb{S}_{k}$ be a nonbinary Boolean semiring, $T: \mathbb{M}_{m, n}\left(\mathbb{S}_{k}\right) \rightarrow$ $\mathbb{M}_{m, n}\left(\mathbb{S}_{k}\right)$ be a linear map. Then $T$ strongly preserves the set $\mathcal{T}_{\text {sa }}\left(\mathbb{S}_{k}\right)$ if and only if $T$ is a $(P, Q, B)$-operator, where $P$ and $Q$ are permutation matrices of appropriate sizes.

Proof. $(\Leftarrow)$ By Lemma 3.2, $(P, Q, B)$-operator preserves the term rank. Hence it strongly preserves the set $\mathcal{T}_{s a}\left(\mathbb{S}_{k}\right)$ as we see in the proof of Theorem 3.3.

$\Rightarrow$ Suppose that $T$ strongly preserves $\mathcal{T}_{s a}\left(\mathbb{S}_{k}\right)$ and $\mathbb{S}_{k}$ is finite with identity 1. By Lemma 3.1, there is some positive integer $d$ such that for $L=T^{d}$, $L^{2}=L$. It is easy to see that $L$ strongly preserves $\mathcal{T}_{s a}\left(\mathbb{S}_{k}\right)$.

Suppose that there exists $i, 1 \leq i \leq m$, such that $L\left(R_{i}\right)$ is not dominated by $R_{i}$. Then there is a pair of indices $(r, s)$ such that $E_{r, s}$ is not dominated by $R_{i}$ and $L\left(R_{i}\right) \geq E_{r, s}$. Let $L\left(R_{i}\right)=X+a E_{r, s}$ where $x_{r, s}=0$ and $a \neq 0$.

Now,

$$
L\left(a R_{i}\right)=L^{2}\left(a R_{i}\right)=L\left(a\left(L\left(R_{i}\right)\right)\right)=L\left(a\left(X+a E_{r, s}\right)\right)=L\left(a X+a^{2} E_{r, s}\right)
$$


but $a^{2}=a$ and $a+a=a$, so

$$
\begin{aligned}
L\left(a R_{i}\right) & =L\left(a X+a E_{r, s}\right)=L\left(a X+a E_{r, s}+a E_{r, s}\right) \\
& =L\left(a X+a E_{r, s}\right)+L\left(a E_{r, s}\right)=L\left(a R_{i}\right)+L\left(a E_{r, s}\right) .
\end{aligned}
$$

Thus, $t\left(L\left(a R_{i}\right)=t\left(L\left(a R_{I}\right)+L\left(a E_{r, s}\right)\right)\right.$. But, $\left(a R_{i}, a E_{r, s}\right) \in \mathcal{T}_{s a}\left(\mathbb{S}_{k}\right)$ and $L$ strongly preserves $\mathcal{T}_{s a}\left(\mathbb{S}_{k}\right)$, so that $\left(L\left(a R_{i}\right), L\left(a E_{r, s}\right)\right) \in \mathcal{T}_{s a}\left(\mathbb{S}_{k}\right)$. That is $t\left(L\left(a R_{i}\right)\right)+t\left(L\left(a E_{r, s}\right)\right)=t\left(L\left(a R_{i}\right)+L\left(a E_{r, s}\right)\right)=t\left(L\left(a R_{i}\right)\right)$. It follows that $t\left(L\left(a E_{r, s}\right)\right)=0$, and hence that $L\left(a E_{r, s}\right)=O$, a contradiction.

We have established that $L\left(R_{i}\right) \leq R_{i}$ for all $i$. Similarly, $L\left(C_{j}\right) \leq C_{j}$ for all $j$. By considering that $E_{i, j}$ is dominated by both $R_{i}$ and $C_{j}$ we have that $L\left(E_{i, j}\right) \leq E_{i, j}$. Since $\mathcal{S}$ is antinegative, we have that $T$ also maps a cell to a multiple of a cell. That is, $T\left(E_{i, j}\right)$ has only one nonzero entry for all $i, j$, and $T(J)$ has all nonzero entries.

So $T$ induces a permutation, $\sigma$, on the set of subscripts $\{1,2, \ldots, m\} \times$ $\{1,2, \ldots, n\}$. That is, $T\left(E_{i, j}\right)=b_{i, j} E_{\sigma(i, j)}$ for some scalars $b_{i, j}$. But $T$ does not preserve term rank if $b_{i, j}$ is zero or a zero-divisor. Moreover we can show that $\mathrm{T}$ maps lines to lines by repeating the arguments used in the proof of Theorem 3.3. Therefore we obtain that $T$ is a $(P, Q, B)$-operator.

Theorem 3.5. Let $\mathbb{S}_{k}$ be a nonbinary Boolean semiring, $T: \mathbb{M}_{m, n}\left(\mathbb{S}_{k}\right) \rightarrow$ $\mathbb{M}_{m, n}\left(\mathbb{S}_{k}\right)$ be a surjective linear map. Then $T$ preserves the set $\mathcal{T}_{\text {sm }}\left(\mathbb{S}_{k}\right)$ if and only if $T$ is a $(P, Q)$-operator, where $P$ and $Q$ are permutation matrices of appropriate sizes.

Proof. If $T$ is surjective, then by Theorem 2.13 we have that $T\left(E_{i, j}\right)=E_{\sigma(i, j)}$ for all $i, j, 1 \leq i \leq m, 1 \leq j \leq n$, where $\sigma$ is a permutation on the set of pairs $(i, j)$.

Suppose that the images of two cells are not in the same line, but the cells are, say $E_{i, j}, E_{i, l}$ are the cells such that $T\left(E_{i, j}\right), T\left(E_{i, l}\right)$ are not in the same line, i.e., $t\left(T\left(E_{i, j}+E_{i, l}\right)\right)=2$. Then $\left(E_{i, j}, E_{i, l}\right) \in \mathcal{T}_{s m}\left(\mathbb{S}_{k}\right)$ but $\left(T\left(E_{i, j}\right), T\left(E_{i, l}\right)\right) \notin$ $\mathcal{T}_{s m}\left(\mathbb{S}_{k}\right)$, a contradiction. Thus $T^{-1}$ maps lines to lines. By Lemma 2.14 it follows that $T^{-1}$ is a $(P, Q)$-operator where $P$ and $Q$ are permutation matrices of appropriate sizes. Hence, $T$ is also of this type.

Conversely, by Lemma 3.2, any $(P, Q)$-operator preserves the term rank. Thus as we see in the proof of Theorem 3.3, any $(P, Q)$-operator preserves the set $\mathcal{T}_{s m}\left(\mathbb{S}_{k}\right)$.

Theorem 3.6. Let $\mathbb{S}_{k}$ be a nonbinary Boolean semiring, $T: \mathbb{M}_{n}\left(\mathbb{S}_{k}\right) \rightarrow \mathbb{M}_{n}\left(\mathbb{S}_{k}\right)$ be a surjective linear map. Then $T$ preserves the set $\mathcal{T}_{m n}\left(\mathbb{S}_{k}\right)$ if and only if $T$ is a nontransposing $\left(P, P^{t}\right)$-operator, where $P$ is a permutation matrix.

Proof. $\quad(\Leftarrow)$ By similar proof of the Lemma 3.2, it is easy to see that any nontransposing $\left(P, P^{t}\right)$-operator preserves $t(A), c(A)$ and $r(A)$. Therefore any nontransposing $\left(P, P^{t}\right)$-operator preserves the set $\mathcal{T}_{m n}\left(\mathbb{S}_{k}\right)$.

$(\Rightarrow)$ Assume that $T$ preserves the set $\mathcal{T}_{m n}\left(\mathbb{S}_{k}\right)$. Since $T$ is surjective, by Theorem 2.13 one has that $T\left(E_{i, j}\right)=E_{\sigma(i, j)}$. 
Let us show that $T$ transforms lines to lines. For all $k$ one has that $\left(E_{i, j}, E_{j, k}\right)$ $\in \mathcal{T}_{m n}\left(\mathbb{S}_{k}\right)$ since

$$
t\left(E_{i, j} E_{j, k}\right)=t\left(E_{i, k}\right)=1=\min \left\{r\left(E_{i, j}\right), c\left(E_{j, k}\right)\right\} .
$$

Thus $\left(T\left(E_{i, j}\right), T\left(E_{j, k}\right)\right) \in \mathcal{T}_{m n}\left(\mathbb{S}_{k}\right)$ by assumption, so

$$
t\left(T\left(E_{i, j}\right) T\left(E_{j, k}\right)\right)=\min \left\{r\left(T\left(E_{i, j}\right)\right), c\left(T\left(E_{j, k}\right)\right)\right\}=1
$$

since $T$ transforms cells to cells. But $T\left(E_{i, j}\right) T\left(E_{j, k}\right)=E_{\sigma(i, j)} E_{\sigma(j, k)}$ so that $E_{\sigma(j, k)}$ is in the same row as $E_{\sigma(j, 1)}$ for every $k$. That is, $T$ maps rows to rows. Similarly $T$ maps columns to columns. That is, $T(X)=P X Q$ for some permutation matrices $P$ and $Q$.

Therefore, $T\left(E_{i, j}\right)=E_{\sigma(i), \tau(j)}$ where $\sigma$ is the permutation corresponding to $P$ and $\tau$ is the permutation corresponding to $Q^{t}$. But, $\left(E_{1, i}, E_{i, 1}\right) \in \mathcal{T}_{m n}\left(\mathbb{S}_{k}\right)$. Thus $\left(E_{\sigma(1), \tau(i)}, E_{\sigma(i), \tau(1)}\right) \in \mathcal{T}_{m n}\left(\mathbb{S}_{k}\right)$ by assumption, and hence $\tau \equiv \sigma$. This implies that $Q^{t}=P$ and hence $T$ is a nontransposing $\left(P, P^{t}\right)$-operator.

To study linear preservers of the equality in the multiplicative low bound the following reduction is vital:

Lemma 3.7. Let $\mathbb{S}_{k}$ be a nonbinary Boolean semiring, and $T: \mathbb{M}_{n}\left(\mathbb{S}_{k}\right) \rightarrow$ $\mathbb{M}_{n}\left(\mathbb{S}_{k}\right)$ preserve the set $\mathcal{T}_{m a}\left(\mathbb{S}_{k}\right)$. Then $T$ preserves the set of matrices with term rank $n$.

Proof. Let $A=0$ and let $B$ be any matrix of term rank $n$ over $\mathbb{S}_{k}$. Then, $t(A)=0, t(A B)=0$. Hence, $t(A B)=t(A)+t(B)-n$. It follows that $t(T(A) T(B))=t(T(A))+t(T(B))-n$. That is $0=0+t(T(B))-n$. It follows that $t(T(B))=n$. That is, $T$ preserves term rank $n$.

Lemma 3.8. Let $\mathbb{S}_{k}$ be a nonbinary Boolean semiring, and $T: \mathbb{M}_{n}\left(\mathbb{S}_{k}\right) \rightarrow$ $\mathbb{M}_{n}\left(\mathbb{S}_{k}\right)$ be a surjective linear map. Then $T$ preserves the set of matrices with term rank $n$ if and only if $T$ is a $(P, Q)$-operator where $P$ and $Q$ are permutation matrices of order $n$.

Proof. $(\Leftarrow)$ By Lemma 3.2, any $(P, Q)$-operator preserves all the term ranks. Thus $T$ preserves the set of matrices with term rank $n$.

$(\Rightarrow)$ By Theorem 2.13 one has that $T\left(E_{i, j}\right)=E_{\sigma(i, j)}$ for all $i, j, 1 \leq i, j \leq n$, where $\sigma$ is a permutation on the set of pairs of indexes. Let us show that $T^{-1}$ maps lines to lines. Assume that the pre-image of a row is not dominated by any line. Then there are indexes $i, k, l$ such that $T^{-1}\left(E_{i, k}\right)$ and $T^{-1}\left(E_{i, l}\right)$ are not in one line. That is, there is indexes $p, r, q, s, p \neq r, q \neq s$, such that $T^{-1}\left(E_{i, k}+E_{i, l}\right) \leq E_{r, s}+E_{p, q}$, and $T^{-1}\left(E_{i, k}+E_{i, l}\right)$ is not dominated by each of the cells $E_{r, s}, E_{p, q}$. By extending $E_{r, s}+E_{p, q}$ to a permutation matrix by adding $n-2$ cells, we find a matrix $A$ such that $t(A)=n$. Since $T$ preserves term rank $n$ by assumption, one has that $t(T(A))=n$. On the other hand, $T(A)$ is dominated by $(n-1)$ lines since $T\left(E_{r, s}\right)=E_{i, k}$ and $T\left(E_{p, q}\right)=E_{i, l}$ lie in one row. This is a contradiction with $t(T(A))=n$. Thus the pre-image of every row is a row or a column. Similarly, the pre-image of every column is a row or a column. It follows by Lemma 2.14 that $T$ is a $(P, Q)$-operator. 
Theorem 3.9. Let $\mathbb{S}_{k}$ be a nonbinary Boolean semiring, $T: \mathbb{M}_{n}\left(\mathbb{S}_{k}\right) \rightarrow \mathbb{M}_{n}\left(\mathbb{S}_{k}\right)$ be a surjective linear operator. Then $T$ preserves the set $\mathcal{T}_{m a}\left(\mathbb{S}_{k}\right)$ if and only if $T$ is a nontransposing $\left(P, P^{t}\right)$-operator, where $P$ is a permutation matrix of order $n$.

Proof. $(\Leftarrow)$ Let us prove that a nontransposing $\left(P, P^{t}\right)$-operator preserve the set $\mathcal{T}_{m a}\left(\mathbb{S}_{k}\right)$. By Lemma 3.2 any $(P, Q)$-operator preserves all the term ranks. Thus the right-hand side of the equality determining $\mathcal{T}_{m a}\left(\mathbb{S}_{k}\right)$ is not changed under the mapping by a nontransposing $\left(P, P^{t}\right)$-operator $T$ and the left-hand side of the equality also is not changed since $t(T(X) T(Y))=t\left(P X P^{t} P Y P^{t}\right)=$ $t\left(P X Y P^{t}\right)=t(X Y)$.

$(\Rightarrow)$ Assume that $T$ preserves the set $\mathcal{T}_{m a}\left(\mathbb{S}_{k}\right)$. Then by Lemma $3.7 T$ preserves the set of matrices with term rank $n$. Since $T$ is surjective, by applying Lemma 3.8 we obtain that $T$ is a $(P, Q)$-operator.

Now, let us see that transposition transformation does not preserve the set $\mathcal{T}_{m a}\left(\mathbb{S}_{k}\right)$. Indeed, the pair $\left(X=E_{i, j}, Y=I-E_{j, j}\right) \in \mathcal{T}_{m a}\left(\mathbb{S}_{k}\right)$ since $t(X Y)=t(0)=0=1+(n-1)-n=t(X)+t(Y)-n$. However, $\left(X^{t}=\right.$ $\left.E_{j, i}, Y^{t}=I-E_{j, j}\right) \notin \mathcal{T}_{m a}\left(\mathbb{S}_{k}\right)$ since $t\left(X^{t} Y^{t}\right)=t\left(E_{j, i}\right)=1 \neq 0$.

It remains to prove that $P Q=I$ the identity matrix. Let us assume that a nontransposing $(P, Q)$-operator preserves the set $\mathcal{T}_{m a}\left(\mathbb{S}_{k}\right)$. Thus one has that $t(X Y)=t(P X Q P Y Q)=t\left((X Q P Y)\right.$ for all pairs $(X, Y) \in \mathcal{T}_{m a}\left(\mathbb{S}_{k}\right)$. The matrix $Q P$ is permutation matrix as a product of two permutation matrices. Assume that $Q P$ permutes $i$ 'th and $j$ 'th columns of $X$. Let $X=E_{i, i}, Y=$ $\sum_{j \neq i} E_{j, j}$. Thus $t(X)=1, t(Y)=n-1, t(X Y)=t(0)=0=t(X)+t(Y)-n$, i.e., $(X, Y) \in \mathcal{T}_{m a}\left(\mathbb{S}_{k}\right)$. On the other side, $X Q P=E_{i, j}$. Thus $X Q P Y=$ $E_{i, j} \neq 0$. Hence, $(T(X), T(Y))=(P X Q, P Y Q) \notin \mathcal{T}_{m a}\left(\mathbb{S}_{k}\right)$. This contradiction concludes that $Q P=I$ and hence $T$ is a nontransposing $\left(P, P^{t}\right)$-operator.

Theorem 3.10. Let $\mathbb{S}_{k}$ be a nonbinary Boolean semiring, and $T: \mathbb{M}_{n}\left(\mathbb{S}_{k}\right) \rightarrow$ $\mathbb{M}_{n}\left(\mathbb{S}_{k}\right)$ be a surjective linear map. Then $T$ preserves the set $\mathcal{T}_{m t}\left(\mathbb{S}_{k}\right)$ if and only if $T$ is a nontransposing $\left(P, P^{t}\right)$-operator where $P$ is a permutation matrix of order $n$.

Proof. $(\Leftarrow)$ By Lemma 3.2, any $(P, Q)$-operator preserves all the term ranks. Thus as we see in the proof of Theorem 3.3 , any nontransposing $\left(P, P^{t}\right)$ operator preserves the set $\mathcal{T}_{m t}\left(\mathbb{S}_{k}\right)$.

$(\Rightarrow)$ By Theorem 2.13 one has that $T\left(E_{i, j}\right)=E_{\sigma(i, j)}$ for all $i, j, 1 \leq i, j \leq n$, where $\sigma$ is a permutation on the set of pairs of indices.

It can be directly checked that $\left(E_{i, j}, E_{j, k}, E_{k, l}\right) \in \mathcal{T}_{m t}\left(\mathbb{S}_{k}\right)$ for all $l$ and for arbitrary fixed $i, j, k$. Thus

$$
\begin{aligned}
& t\left(T\left(E_{i, j}\right) T\left(E_{j, k}\right)\right)+t\left(T\left(E_{j, k}\right) T\left(E_{k, l}\right)\right) \\
= & t\left(T\left(E_{i, j}\right) T\left(E_{j, k}\right) T\left(E_{k, l}\right)\right)+t\left(T\left(E_{j, k}\right)\right) .
\end{aligned}
$$

Let us denote $T\left(E_{i, j}\right)=E_{p, q}, T\left(E_{j, k}\right)=E_{r, s}$, and $T\left(E_{k, l}\right)=E_{u, v}$. Since $t\left(E_{r, s}\right)=1 \neq 0$, it follows from the equality (1) that either $q=r$ or $s=u$ or 
both. If for all $l=1, \ldots, n$ it holds that $q=r$ or for all $l=1, \ldots, n$ it holds that $s=u$, then it is easy to see that $T$ maps lines to lines. Assume that there exists an index $l$ such that $r \neq q$. Thus by (1) $s=u$. Hence, for arbitrary $m$, $1 \leq m \leq n$ one has that $\left(E_{i, j}, E_{j, k}, E_{k, m}\right) \in \mathcal{T}_{m t}\left(\mathbb{S}_{k}\right)$. Denote, $T\left(E_{k, m}\right)=E_{w, z}$. Using the previous notations, one obtains that $\left(E_{p, q}, E_{r, s}, E_{w, z}\right) \in \mathcal{T}_{m t}\left(\mathbb{S}_{k}\right)$. Since $q \neq r$ it follows that $w=s$ and hence $\mathrm{T}$ maps $k$ th row to $s$ th row. Thus in this case we obtain that rows are transformed to rows. By the same arguments with the first matrix it is easy to see that columns are transformed to columns. In the other case $s \neq u$ and $q=r$ one obtains that rows are transformed to columns and columns to rows.

By Lemma 2.14 it follows that there exists a permutation matrices $P$ and $Q$ such that $T(X)=P X Q$ for all $X \in \mathbb{M}_{n}\left(\mathbb{S}_{k}\right)$ or $T(X)=P X^{t} Q$.

In order to show that the transposition transformation does not preserve $\mathcal{T}_{m t}\left(\mathbb{S}_{k}\right)$ it suffices to note that $\left(E_{i, j}, I, I-E_{j, j}\right) \in \mathcal{T}_{m t}\left(\mathbb{S}_{k}\right)$ and $\left(E_{j, i}, I, I-\right.$ $\left.E_{j, j}\right) \notin \mathcal{T}_{m t}\left(\mathbb{S}_{k}\right)$.

In order to show that $Q=P^{t}$ it suffices to note that $\left(E_{i, j}, E_{j, j}, E_{j, i}\right) \in$ $\mathcal{T}_{m t}\left(\mathbb{S}_{k}\right)$. Denote that $T\left(E_{i, j}\right)=E_{\sigma(i), \tau(j)}$ where $\sigma$ is the permutation corresponding to $P$ and $\tau$ is the permutation corresponding to $Q^{t}$. Therefore, $\left(E_{\sigma(i), \tau(j)}, E_{\sigma(j), \tau(j)}, E_{\sigma(j), \tau(i)}\right) \in \mathcal{T}_{m t}\left(\mathbb{S}_{k}\right)$ by assumption, and hence $\tau \equiv \sigma$. This implies that $Q^{t}=P$ and hence $T$ is a nontransposing $\left(P, P^{t}\right)$-operator.

As a concluding remark, we have characterized the linear operators that preserve the extreme sets of matrix ordered pairs over nonbinary Boolean semirings which come from certain term rank inequalities over nonbinary Boolean semirings.

\section{References}

[1] L. B. Beasley, A. E. Guterman, Rank inequalities over semirings, J. Korean Math. Soc. 42 (2005), no. 2, 223-241.

[2] _ Linear preservers of extremes of rank inequalities over semirings, J. Math. Sciences 131 (2005), no. 5, 5919-5938.

[3] L. B. Beasley, A. E. Guterman, Y. B. Jun, and S. Z. Song, Linear preservers of extremes of rank inequalities over semirings: row and column ranks, Linear Algebra and Its Applications, 413 (2006), no. 2-3, 495-509.

[4] L. B. Beasley, S. G. Lee, and S. Z. Song, Linear operators that preserve pairs of matrices which satisfy extreme rank properties, Linear Algebra Appl. 350 (2002), 263-272.

[5] L. B. Beasley and N. J. Pullman, Term rank, permanent and rook polynomial preservers, Linear Algebra Appl. 90 (1987), 33-46.

[6] L. B. Beasley, S. Z. Song, and K. T. Kang, Preservers of term ranks of symmetric matrices, Linear Algebra Appl. 436 (2012), no. 6, 1727-1738.

[7] K. T. Kang, S. Z. Song, and L. B. Beasley, Linear preservers of term ranks of matrices over semirings, Linear Algebra Appl. 436 (2012), no. 7, 1850-1862.

[8] S. Kirkland and N. J. Pullman, Linear operators preserving invariants of nonbinary matrices, Linear and Multilinear Algebra 33 (1992), no. 3-4, 295-300.

[9] C. K. Li and S. Pierce, Linear preserver problems, Amer. Math. Monthly 108 (2001), no. $7,591-605$. 
[10] S. Pierce and others, A survey of linear preserver problems contents, Linear and Multilinear Algebra 33 (1992), no. 1-2, 1-119.

[11] S. Z. Song and M. H. Kang, Linear Preservers of Extremes of Matrix Pairs Over Nonbinary Boolean Algebra, Bull. Malays. Math. Sci. Soc. 36 (2013), no. 1, 73-81.

[12] S. Z. Song, M. H. Kang, and Y. B. Jun, Extreme sets of rank inequalities over Boolean matrices and their preservers, Commun. Korean Math. Soc. 28 (2013), no. 1, 1-9.

LERoy B. BEASLEY

Department of Mathematics and Statistics

Utah State University

LOGAN, UTAH 84322-3900, USA

E-mail address: leroy.b.beasley@aggiemail.usu.edu

SEONG-HEE HEO

Department of Mathematics

Jeju National University

JEJU 690-756, KOREA

E-mail address: hsh33089@daum.net

SEOK-Zun SONG

Department of Mathematics AND

Research Institute For Basic Sciences

Jeju National University

JEJU 690-756, KOREA

E-mail address: szsong@jejunu.ac.kr 